\title{
Aspects of Slow-Wave EEG Activity During Sleep in Twins Discordant for Chronic Fatigue Syndrome
}

\author{
Robert Hoffmann*,1, Jack Goldberg ${ }^{2}$, Nathaniel F. Watson ${ }^{3}$, Dedra Buchwald ${ }^{4}$ and Roseanne Armitage ${ }^{1}$ \\ ${ }^{I}$ Department of Psychiatry, University of Michigan, Ann Arbor, MI, USA; and the Departments of ${ }^{2}$ Epidemiology, \\ ${ }^{3}$ Neurology, ${ }^{4}$ Medicine, University of Washington, Seattle, WA, USA
}

\begin{abstract}
Chronic Fatigue Syndrome (CFS) is a disease characterized by high levels of daytime fatigue. Complaints such as unrefreshing sleep and insomnia are common. Polygraphic studies of sleep in CFS patients have found evidence of disturbed sleep, but controls in these studies were often not adequate to evaluate differences specific to CFS. Watson, et al. [1] and Ball, et al. [2] used a co-twin control design to eliminate virtually all sources of confounding variance and found a small difference between levels of Stage 2 sleep (lower in CFS) and Stage 3 sleep (higher in CFS), where CFS ill twins showed a higher level of sleep complaint than the healthy twins which was not reflected in their sleep physiology. The current study will apply more sensitive measures of sleep EEG to these data to examine differences more closely. A cotwin control study was performed on 10 pairs of identical twins discordant for CFS. Data from the second sleep night were analyzed using fast-fourier analysis (FFT) and finally measures of slow-wave activity (SWA). Data for NREM periods was analyzed. There was a significant interaction in SWA for Twin x NREM period for the first 2 NREM periods with the healthy twins having more SWA in the first NREM period and less in the second NREM period. While overall SWA activity did not differentiate the groups, the distribution of SWA in the first 2 NREM periods could be associated with the experience of unrefreshing sleep. Typically, SWA in the first NREM period is associated with deep restorative sleep. Lower SWA in NREMP 1 in the ill CFS twins suggests that this process is compromised resulting in the experience of less refreshing sleep.
\end{abstract}

Keywords: Chronic fatigue syndrome, sleep, power spectral analysis, slow-wave activity, NREM, twins.

\section{INTRODUCTION}

Chronic fatigue syndrome (CFS) is an illness characterized by profound fatigue lasting at least 6 months accompanied by disturbances of sleep and mood, as well as musculoskeletal pain, and other symptoms [3-5]. In addition, CFS patients can have a very high incidence (58\%) of sleep disorders (e.g. apnea/hypopnea index, restless legs syndrome) [6]. While some symptoms are similar to those of patients with Major Depressive Disorder (MDD), CFS itself does not appear to arise from underlying MDD [7]. Also, there is no consensus as to the underlying pathophysiology of the disease [7, 8]. Along with excessive daytime fatigue, symptoms of insomnia and insufficient, non-restorative sleep are among the most common and disabling complaints [4-6, 9-15].

Several studies have looked at polygraph data for CFS patients. Although the results and procedures were varied; sleep efficiency was reduced in 7 of 7 studies [14, 16-21], time in bed was increased in 2 out of 2 studies [17, 18] and wake after sleep onset was increased in 3 out of 3 studies $[17,18,22]$. Control subjects in these studies varied from patients with MDD to co-twin controls. The co-twin control was used by Watson [23] and Ball, et al. [2], where they found an increase in percent stage 3 , a decrease in percent

*Address correspondence to this author at the Sleep \& Chronophysiology Laboratory, University of Michigan, 2101 Commonwealth, Ann Arbor, MI 48105, USA; Tel: 734-764-1320; Fax: 734-764-1229;

E-mail:rhhoffma@umich.edu stage 2 and an increase in percent REM sleep in CFS ill twins. The difference in percent stage 3 was small (ill twins $10.7 \%$ vs healthy twins $8.6 \%$ ), which was statistically significant. This difference was accompanied by a slight reduction in percent stage 2 for the CFS ill twins (ill twins 44.9\%, healthy twins $49.0 \%$ ). When using the standard sleep stage scoring manual [24] the cutoff for changing from stage 2 to stage 3 is the presence of at least $20 \%$ delta activity. A slight elevation in delta amplitude in the CFS patients could account for this difference between the groups. Also, these authors did not find any difference between ill and healthy twins for sleep efficiency. This suggests that the co-twin control design provides a much better assessment of differences due to disease.

There are two studies that looked simultaneously at subjective reports of sleep quality and polygraphic recordings of sleep EEG [23, 25]. In both studies the CFS patients judged their sleep to be bad or unrefreshing. But there were no identifiable group differences in sleep stage profiles. The comparison groups consisted of non-fatigued healthy subjects [25] or co-twin controls [1]. The suggestion here is that perception of the quality of sleep in CFS patients may be altered by the condition. This, however, cannot account for excessive daytime fatigue.

Several articles have suggested that a pattern of alpha/delta activity during non-REM sleep could be a part of the sleep physiology of CFS patients [15, 20,26]. Such EEG patterns have been shown in patients with fibromyalgia [27, 28], but its influence in CFS is still not clear. Manu [20] stated that "Alpha-delta sleep is not a marker of fibromyalgia 
or CFS..." (p.465). Others have reported an association between alpha-delta intrusion and anxiety [26], but there was no control group of healthy subjects in this study for comparison; Whelton [15] compared CFS patients to healthy controls. In this study, CFS patients described unrefreshing sleep and showed non-rem sleep alpha-delta activity, but they did not show signs of physiological daytime sleepiness. This finding together with those of Watson, 2003 and Majer, 2007 suggest that a careful examination of sleep EEG could identify activity resulting in subjective reports of unrefreshing sleep, but would not effect the overall pattern of sleep stages during the night.

Although the exact mechanisms of alterations in sleep physiology in CFS have not been firmly established, changes in sleep homeostatic mechanisms are likely involved. Sleep physiology appears to be controlled by 2 opposing processes - sleep homeostasis or Process S, and circadian drive or Process C [29]. Process S accumulates during wakefulness, dissipates rapidly over non-rapid eye movement (NREM) sleep time, and is reflected in the time course of slow wave activity (SWA) during the night (i.e., delta power or amplitude in NREM sleep). Process C, on the other hand, reflects the circadian timing of REM sleep and the drive for wakefulness [30]. The importance of the homeostatic component of this model is supported by the observed increase in SWA following sleep deprivation [31]. In addition, the amount of prior wakefulness has been monotonically related to an increase in SWA power after acute sleep restriction dissipating quickly over NREM sleep time [32, 33]. Because reduced slow-wave sleep reflects compromised sleep homeostasis in fibromyalgia [34], changes in SWA would suggest a similar homeostatic disruption in CFS.

As many aspects of sleep physiology such as REM density, stage 2 sleep, slow wave sleep, and body movements are genetically determined [35, 36], genetic confounding may obscure the relationship between sleep physiology and CFS. Co-twin control studies, adjusted for genetic and many environmental factors, offer a powerful alternative to traditional approaches that compare CFS patients to healthy individuals [37]. This research design is particularly valuable in the studies of sleep, where genetic factors contribute substantially to sleep architecture [1], the number of data points generated is large, and the range of values observed in normal individuals is wide. We, therefore, compared the power spectral analysis of sleep EEGs between monozygotic twins discordant for CFS and asked 1) Does the amount and progression of slow-wave activity in NREM sleep periods across the night vary according to CFS status? And 2) Does alpha and delta activity occur together during sleep in CFS?

\section{METHODS}

\section{Participants}

From 1997 to 1999,22 sets of CFS discordant twins from the University of Washington CFS Twin Registry were chosen for a 7-day in-person evaluation based on registry information and telephone screening establishing the presence or absence of symptoms consistent with the Centers for Disease Control and Prevention (CDC) diagnostic criteria of CFS [13,23 ]. Twins were required to 1) be at least 18 years of age; 2) be reared together; 3 ) be discordant for CFS (one twin met the CDC CFS criteria, the other did not); 4) abstain from alcohol and caffeine and, based on their personal physicians' advice, discontinue all medications at least 2 weeks prior to the evaluation; and 5) travel to Seattle together.

To determine if a twin met CDC CFS criteria, we used responses to the CFS symptom checklist, diagnoses generated by a structured interview, and information from review of the twins' medical records. Medical records covering the last 5 years were reviewed by a physician knowledgeable about CFS (DB) for exclusionary medical conditions. A psychologist and infectious disease specialist also independently reviewed the twins' medical charts to verify health status and approve twins for participation. Depression was assessed using the Diagnostic Interview Schedule Version III-A [38], with diagnoses based on the Diagnostic and Statistical Manual III [39]. Monozygosity was initially determined using previously validated self-report methods [40, 41], then confirmed with analysis of restriction fragment length polymorphisms. This technique allows probability of monozygosity to be ascertained with $99.9 \%$ certainty [42].

Prior to the scheduled visit, we confirmed that the ill twin still met CFS criteria and that the control twin was healthy. The same inclusion and exclusion criteria (e.g., body mass index, psychiatric disorders) and review processes were applied to both the CFS and healthy twins. Written informed consent was obtained from all twins in accordance with regulations of the University of Washington Institutional Human Subjects Office. A waiver of consent was obtained from the University of Michigan IRB to conduct the quantitative analysis of the sleep EEG.

\section{Polysomnography}

For this analysis, we were able to successfully extract EEG data for quantification on a subset of 10 pairs. Polysomnography with a full recording montage was performed during 2 nights, including central and occipital electroencephalogram, left and right electrooculogram, mental and submental electromyogram, chest and abdominal respiratory effort, nasal and oral airflow, left and right anterior tibialis electromyogram, pulse oximetry, electrocardiogram, body position, and microphone-detected snoring. Data were recorded on an ALICE $3^{\mathrm{TM}}$ digital system (Respironics/ Healthdyne Technologies, Murrysville, PA 15668-8550). Visual polysomnography scoring was performed according to standard Rechtschaffen and Kales criteria by a single technician blinded to illness status [24]. All data were derived from the second night, with the first night being used for acclimatization to the sleep-lab environment. Twins had traveled to Seattle at least 4 nights prior to the acclimatization night and 5 nights prior to the study night. The following sleep-related parameters were calculated: total sleep time, sleep latency, REM latency, sleep staging, sleep efficiency, hypnogram awakenings, arousal number, and arousal index.

\section{EEG Quantification}

Power spectral analysis was performed using standard Fast Fourier Transform software [43] on data sampled at $100 \mathrm{~Hz}$. Each $30 \mathrm{sec}$ epoch of data was analyzed in 2 second blocks that were recombined to provide values for 5 frequency bands: delta $(0.5-4 \mathrm{~Hz})$, theta $(4-8 \mathrm{~Hz})$, alpha $(8-12$ $\mathrm{Hz})$, sigma $(12-16 \mathrm{~Hz})$, and beta $(>16 \mathrm{~Hz})$. This analysis 
Table 1. Selected Demographic and Clinical Characteristics for 10 Pairs of CFS Discordant Twins

\begin{tabular}{|c|c|c|}
\hline Characteristic & CFS Twins & Healthy Twins \\
\hline \hline Marital status, $\%$ & 40 & 60 \\
\hline Post-secondary education, $\%$ & 50 & $27.2(7.9)$ \\
\hline BMI, $k g / m^{2}$ (standard deviation) & $27.3(8.3)$ & 10 \\
\hline Lifetime major depressive disorder, $\%$ & 30 & 0 \\
\hline Current major depressive disorder, $\%$ & 0 & 0 \\
\hline CFS duration, years (standard deviation) & $6.3(5.6)$ & 0 \\
\hline
\end{tabular}

was performed for both left (C3) and right (C4) hemisphere central electrode sites. Data from the two hemispheres was combined for the final analysis. Upon completion, a data set was produced containing sleep stage scores along with the quantified data.

\section{Statistical Analyses}

Fast Fourier Transform data was coded for sleep stage and illness status. Across the nights, NREM periods were identified [44] within which SWA measures were generated. This was done by averaging delta activity for stages 2, 3 and 4 for each NREM period. Data were analyzed both as the value of the power for each NREM period and with the power for each NREM period expressed as a percentage of the average power for the combined NREM periods for that night. We performed a repeated measures ANOVA analysis of SWA for the first 3 NREM periods for the left and right central electrodes combined using TWIN and NREM period as independent variables. To explore the time course for changes in SWA across the night for CFS and healthy twins, the value for SWA for each NREM period was divided by the average SWA to generate a percent SWA measure. This transformation eliminates any overall EEG amplitude differences between subjects by referring to EEG SWA activity as a percentage of the overall EEG SWA for each subject. Statistical analyses were either repeated measures MANOVAs, ANOVAs and t-tests as appropriate. The two independent variables were TWIN (CFS versus healthy) and NREM period (first, second, third). The fourth NREMP was not used in the analyses, since in three pairs of twins at least one failed to show the fourth NREMP, which otherwise would have reduced the power of the statistical analysis.

\section{RESULTS}

The demographic data for the participants in this study are presented in Table 1. Differences were minimal among these identical twins.

Sleep architecture scored by traditional methods are shown in Table 2 . No differences were observed between the CFS and healthy twins.

The means and standard deviations for the analysis of SWA for the first 3 NREM periods are presented in Table 3. The main effect for TWIN was significant $(\mathrm{F}(1,7)=7.31 ; P$ $<0.05)$ but the interaction between TWIN and NREM period $(\mathrm{F}(2,14)=2.76, P=0.0974)$ was not. Post-hoc paired t-tests on these data revealed a significant difference for TWIN at NREM period $2(\mathrm{t}=3.82, \mathrm{df}=9, P<0.005)$.

To explore the time course for changes in SWA across the night among the CFS and healthy twins, SWA for each NREM period was divided by the average SWA to generate a percent SWA measure. The values for this conversion are shown in Fig. (1). The repeated measures ANOVA revealed a non-significant interaction for TWIN versus NREM period $(\mathrm{F}(2,14)=2.91)$; however in Fig. (1) the TWIN difference appears to emerge in the first 2 NREM periods. A repeated measures ANOVA on these 2 points alone yielded a significant 2 way interaction between TWIN and NREM period ( $\mathrm{F}$ $(1,8)=8.81 ; P<0.02)$.

Table 2. Means (Standard Deviations) for Selected Sleep Stage Variables for CFS Discordant Twins

\begin{tabular}{|c|c|c|c|}
\hline Sleep Variable & CFS Twins & Healthy Twins & t value (p) \\
\hline \hline Total Sleep Time, minutes & $391.4(39.1)$ & $396.5(43.8)$ & $-0.78(0.46)$ \\
\hline Sleep Latency, minutes & $14.5(22.1)$ & $10.9(10.0)$ & $0.47(0.65)$ \\
\hline REM Latency, minutes & $67.6(35)$ & $79.1(13.3)$ & $-1.14(0.28)$ \\
\hline Stage 1, \% & $6.9(2.9)$ & $7.1(13.8)$ & $0.32(0.76)$ \\
\hline Stage 2, \% & $49.5(9.5)$ & $54.1(7.7)$ & $1.8(0.11)$ \\
\hline Stage 3, \% & $12.2(7.1)$ & $7.2(4.9)$ & $3.180(0.01)$ \\
\hline Stage 4, \% & $2.8(4.3)$ & $2.4(5.4)$ & $0.39(0.70)$ \\
\hline REM, \% & $33.8(9.2)$ & $31.6(3.4)$ & $0.83(0.43)$ \\
\hline
\end{tabular}


Table 3. Means (Standard Deviations) for Slow Wave Activity for the First 3 NREM Periods Among 10 Pairs of CFS Discordant Twins

\begin{tabular}{|c|c|c|c|}
\hline Slow Wave Activity Variable & CFS Twins & Healthy Twins & t value (p) \\
\hline \hline NREM 1 & $535.9(117.6)$ & $522.5(149.3)$ & $10.4(14.6)$ \\
Latency, minutes & $9.9(9.0)$ & $67.9(28.4)$ & $0.42(.69)$ \\
Duration, minutes & $48.8(22.5)$ & $406.1(72.2)$ & $3.82(.005)$ \\
\hline NREM 2 & $505.7(128.9)$ & $112.7(32.7)$ & \\
Latency, minutes & $87.3(38.8)$ & $66.7(34.6)$ & \\
Duration, minutes & $61.1(32.2)$ & $356.1(77.9)$ & \\
NREM 3 & $457.1(148.9)$ & $208.6(69.6)$ & \\
Latency, minutes & $192.8(83.6)$ & $59.0(19.7)$ & \\
\hline
\end{tabular}

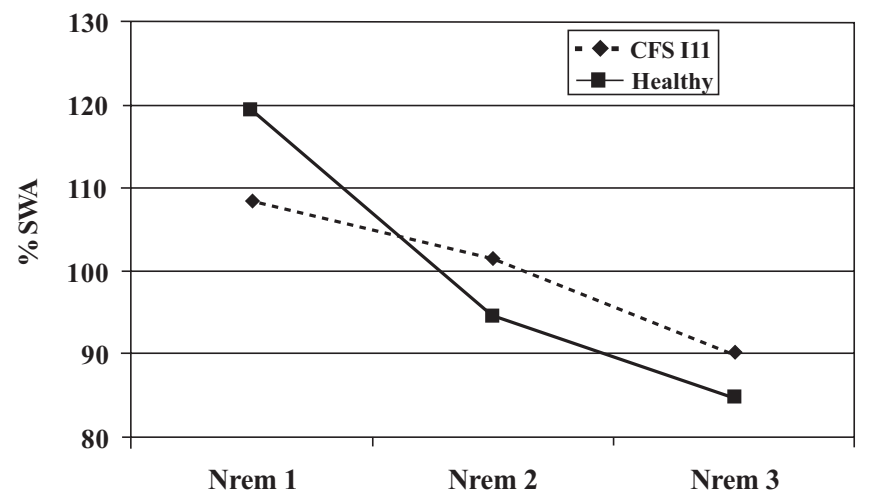

Fig. (1). Percent slow wave sleep activity across first 3 NREM periods for CFS and healthy twins. NREM period; $\mathrm{F}(2,14)=5.95$; $P<0.02)$; Twin $*$ NREM period; F $(2,14)=2.91$; n.s.; NREM periods 1 and $2 ; \mathrm{F}(1,8=4.87 ; \mathrm{p}=.03)$.

Exponential curves were fit to the \%SWA activity for the two twin groups using the equation $y=b * e^{c * \text { time }}$ where $b$ is the expected SWA power at time 0, $\mathrm{c}$ is the exponential change or decay, and time is the minutes of NREM sleep since sleep onset [45]. The y intercepts reflecting asymptotic power were 123.2 for the ill twins and 132.2 for the healthy twins. The exponents for the two groups (ill vs healthy) were -.00120 and -.00149 respectively. All means fall within the 95\% confidence level for the groups; therefore these groups did not differ statistically. Qualitatively, the exponential values do conform to the overall evaluation of a flatter course of SWA activity decline across NREMPs in the ill twins, and a greater buildup of Process $S$ in the healthy twins (see Fig. 1). Intra-class correlations were applied to the exponential yielding a correlation of $0.6474(\mathrm{p}<.02)$ for the exponentials and $0.7232(\mathrm{p}<.004)$ for the $y$ intercepts showing a high degree of concordance between each pair of twins.

Correlations were generated for amounts of alpha and delta activity during sleep in each twin. The average of these values did not differ between the CFS ill and healthy twins $(0.612 \pm 0.217$ versus $0.655 \pm 0.161, t=0.96, d f=9, p=.36)$. In addition, the average amount of alpha activity for the CFS ill and healthy twins was also similar $(87.0 \pm 39.6$ versus $82.1 \pm 35.2, \mathrm{t}=1.60, \mathrm{df}=9, \mathrm{p}=.14$.

\section{DISCUSSION}

This study of SWA in CFS was particularly relevant given the hallmark complaints of severe fatigue and postexertional fatigue in CFS and the association between measures of SWA and recovery from fatigue shown in previous studies [33, 46]. Although we demonstrated some small but statistically significant differences in SWA between CFS twins and their healthy co-twins, neither the sleep stage data (a slight elevation in percent stage 3 sleep) nor the quantified SWA data showed differences of a magnitude congruent with the striking fatigue complaints of the CFS twins. Even so, our findings offer some insight into potential subtle differences in sleep physiology in CFS. For example, the amount of SWA across all NREM periods, although similar, did deviate from equality, an evaluation of NREM periods did reveal a trend $(\mathrm{F}(1,8)=4.87 ; \mathrm{p}=.06)$. Looking at NREM periods 1 and 2 separately showed a significant difference between groups for NREM period $2(\mathrm{~F}(2,14)=5.95 ; P<$ 0.02 ). Because elevated levels of fatigue generated by sleep deprivation are associated with elevated SWA in NREM period 1 [33], the sleep of the severely fatigued twins was unexpected.

The intercepts for the ill and healthy twins were 123.2 and 132.2 respectively. When all twins were used in one analysis, the intercept was 127.1. Armitage (2000) [47] performed a similar analysis on data from healthy women with no personal or family history of psychopathology and reported an intercept of 119.1 (95\% confidence interval 111.3126.7). Both the CFS ill and well twins had higher asymptotic SWA, outside the $95 \%$ confidence interval for the healthy control women, but with a significantly slower rate of decay. These findings do suggest that while the total amount of SWA may not be reduced in those with CFS or their identical twins, the time course of SWA is outside the normative ranges in healthy women.

According to the Process $\mathrm{S}$ theory of sleep regulation, drive for SWA accumulates during the awake hours prior to sleep. SWA should be maximal in the first NREM sleep episode and should dissipate quickly. Sleep deprivation or sleep delay [33] will enhance SWA and is associated with a faster dissipation. Lower asymptotic SWA may reflect a failure to accumulate sleep need during wakefulness, whereas a slower 
rate of decay may reflect a failure to recover or fulfill sleep debt. Our findings in CFS suggest that neither the ill nor the well twin showed a failure to accumulate sleep need, as asymptotic SWA power was higher than healthy control women. However, the decay rate or dissipation of SWA was significantly slower in both the CFS ill and CFS well twins compared to published data from healthy control women. The present study included only baseline sleep data and therefore is merely suggestive of sleep regulatory impairment. A more definitive test would be to examine the SWA response to sleep challenge in the CFS twins. We recently conducted such a study, including 5 pairs of the CFS twins reported here, and demonstrated that the SWA response to challenge was significantly lower with a slower dissipation in the CFS ill twins [45]. Taking the results of the two studies together, it does appear that while some aspects of SWA may be outside the normal limits in both the CFS ill and well twins, it is only the ill twins who showed abnormalities in sleep regulation.

According to standard sleep stage scoring [24], the transition from waking to sleep is marked by a cessation of the generation of alpha activity. If the alpha activity does not cease, this may indicate that the brain is not totally 'asleep', thereby accounting for poor recuperative sleep. A cooccurrence of alpha activity during NREM sleep was shown by Moldofsky, et al. 1975 [48] in patients with fibrositis and by Roizenblatt, et al. 2001 [27] in patients with fibromyalgia. Their research showed alpha activity during NREM sleep episodes, where delta is the prominent EEG frequency. This was interpreted as an elevation of CNS activity towards a more aroused state thus generating daytime fatigue. In the current study the correlation of alpha and delta activity during sleep, or in the amounts of alpha generated during sleep, between the 2 groups of twins, was not significantly different. The use of the co-twin control design makes this study an extremely sensitive test of the alpha/delta hypothesis, as a possible mechanism for the daytime fatigue observed in CFS patients.

This study, however, has several limitations. First, as only 10 pairs of twins were examined our statistical power to detect differences in sleep physiology in the CFS discordant twins may be limited, and our results may not be replicated in another sample of CFS-discordant twins. The CFS twins in the study were reared together while a better evaluation of true genetic contribution would come from twins reared apart. Such a group would allow an investigation of genetic and environment contributions to differences between twins.

\section{CONCLUSIONS}

In summary, while standard metrics used to assess fatigue and sleep may not be adequate and to distinguish CFS ill and healthy twins, computer quantification of sleep EEG was used to reveal underpinnings of the prominent sleep complaints in CFS. This alone is not enough to completely explain the cause of excessive daytime fatigue in CFS ill patients, but it does point to differences in sleep physiology that may contribute to excessive daytime fatigue. Although more sensitive quantitative measures of sleep EEG suggest a problem with the recuperative function of sleep in CFS, further work is necessary to understand this phenomenon. Future research should analyze data from larger groups of par- ticipants or bring revised theories of physiological markers of fatigue to the area of CFS. Given our results, clinical intervention should be directed at improving the quality of sleep in patients with CFS. Their complaints are similar to patients with insomnia, therefore treatment for that may have some efficacy in patients with CFS. Paradoxically, behavioral treatment for insomnia starts with sleep restriction. This may help the patient by forcing a restructuring of sleep to be more efficient which would improve the problems we have identified here.

\section{ACKNOWLEDGEMENTS}

Research supported by NIH U19A138429 (Dr. Buchwald) and the Center for Women's Health Gender Research NR04011 (Drs. Landis and Lentz). This study would not have been completed without the continuous contribution of Roseanne Armitage. We also like to thank the twins who participated in the CFS Twin Registry, and those who visited Seattle to conduct this research.

\section{REFERENCES}

[1] Watson NF, Jacobsen C, Goldberg J, Kapur V, Buchwald D. Subjective and objective sleepiness in monozygotic twins discordant for chronic fatigue syndrome. Sleep 2004; 27: 973-7.

[2] Ball N, Buchwald DS, Schmidt D, Goldberg J, Ashton S, Armitage R. Monozygotic twins discordant for chronic fatigue syndrome: objective measures of sleep. J Psychosom Res 2004; 56: 207-12.

[3] Fukuda K, Straus SE, Hickie I, Sharpe MC, Dobbins JG, Komaroff A. The chronic fatigue syndrome: a comprehensive approach to its definition and study. International Chronic Fatigue Syndrome Case Definition Study Group. Ann Intern Med 1994; 121: 953-9.

[4] Komaroff AL, Buchwald D. Symptoms and signs of chronic fatigue syndrome. Rev Infect Dis 1991; 13: S8-11.

[5] Komaroff AL, Buchwald DS. Chronic fatigue syndrome: An update. In: Coggins C, Hancock E, Levitt L, Eds. Annual Review of Medicine. Palo Alt, USA: Annual Reviews 1998; vol. 49: pp. 1-13.

[6] Fossey M, Libman E, Bailes S, et al. Sleep quality and psychological adjustment in chronic fatigue syndrome. J Behav Med 2004; 27(6): 581-601.

[7] Fischler B. Review of clinical and psychobiological dimensions of the chronic fatigue syndrome: differencitation from depression and contribution of sleep dysfunctions. Sleep Med Rev 1999; 3(2): 131 46.

[8] Maquet D, Demoulin D, Crielaard J. Chronic fatigue syndrome: a systemic review. Ann Readapt Med Phys 2006; 49(6): 418-27.

[9] Neu D, Mairesse O, Hoffmann G, et al. Sleep quality perception in the chronic fatigue syndrome. Correlations with sleep efficiency, affective symptoms and intensity of fatigue. Neuropsychobiology 2007; 56: 40-6.

[10] Schaefer K. Sleep disturbances and fatigue in women with fibromyalgia and chronic fatigue syndrome. J Obstet Gynecol Neonatal Nurs 1995; 24(3): 229-33.

[11] Morriss RK, Wearden AJ, Battersby L. The relation of sleep difficulties to fatigue, mood and disability in chronic fatigue syndrome. J Psychosom Res 1997; 42(6): 597-605.

[12] Unger E, Nisenbaum R, Moldofsky H, et al. Sleep assessment in a population-based study of chronic fatigue syndrome. BMC Neurol 2004; 4(6): doi: 10.1186/1471-2377-4-6.

[13] Buchwald D, Pascualy R, Bombardier C, Kith P. Sleep disorders in patients with chronic fatigue. Clin Infect Dis 1994; 18(suppl 1): S68-72.

[14] Krupp LB, Jandorf L, Coyle PK, Mendelson WB. Sleep disturbance in chronic fatigue syndrome. J Psychosom Res 1993; 37(4): 325-31.

[15] Whelton CL, Salit I, Moldofsky H. Sleep, Epstein-Barr virus infection, musculoskeletal pain, and depressive symptoms in chronic fatigue syndrome. J Rheumatol 1992; 19: 939-43.

[16] Fischler B, LeBon O, Hoffmann G, Cluydts R, Kaufman L, DeMeirleir K. Sleep anomalies in the chronic fatigue syndrome. A comorbidity study. Neuropsychobiology 1997; 35(3): 115-22. 
[17] Sharpley A, Clements A, Hawton K, Sharpe M. Do patients with "pure" chronic fatigue syndrome (neurasthenia) have abnormal sleep? Psychosom Med 1997; 59: 592-6.

[18] Morriss R, Sharpe M, Sharpley AL, Cowen P, Hawton K, Morris J. Abnormalities of sleep in patients with the chronic fatigue syndrome. Br J Med 1993; 306: 1161-4.

[19] Guilleminault C, Poyares D, Rosa A, Kirisoglu C, Almeida T, Lopes MC. Chronic fatigue, unrefreshing sleep and nocturnal polysomnography. Sleep Med 2006; 7: 513-20.

[20] Manu P, Lane TJ, Matthews DA, Castriotta RJ, Watson RK, Abeles M. Alpha-delta sleep in patients with a chief complaint of chronic fatigue. Southern Med J 1994; 87(4): 465-70.

[21] Zubieta JK, Demitrack MA, Shipley JE, Engleberg ND, Eiser A, Douglas A. Sleep EEG in chronic fatigue syndrome: comparison with major depression. Biol Psycol 1993; 33: 73A.

[22] Stores G, Fry A, Crawford C. Sleep abnormalities demonstrated by home polysomnography in teenagers with chronic fatigue syndrome. J Psychosom Res 1998; 45(1): 85-91.

[23] Watson NF, Kapur V, Arguelles LM, et al. Comparison of subjective and objective measures of insomnia in monozygotic twins discordant for chronic fatigue syndrome. Sleep 2003; 26: 324-8.

[24] Rechtschaffen A, Kales A. A manual of standardized terminology, techniques and scoring system for sleep stages of human subjects. National Institutes of Health Publication No. 204 ed. In: Berger RJ, Dement WC, Jacobson A, et al., Eds. Washington, D.C.: Public Health Service, U.S. Government Printing Office 1968.

[25] Majer M, Jones J, Unger E, et al. Perception versus polysomnographic assessment of sleep in CFS and non-fatigued control subjects: results from a population-based study. BMC Neurol 2007; 7: 40.

[26] VanHoof E, DeBecker P, Lapp C, Cluydts R, DeMeirleir K. Defining the occurrence and influence of alpha-delta sleep in chronic fatigue syndrome. Am J Med Sci 2007; 333(2): 78-84.

[27] Roizenblatt S, Moldofsky H, Benedito-Silva AA, Tufik S. Alpha sleep characteristics in fibromyalgia. Arthritis Rheum 2001; 44: 222-30.

[28] Moldofsky H. Sleep and pain. Sleep Med Rev 2001; 5(5): 387-98.

[29] Borbély A. A two-process model of sleep regulation. Hum Neurobiol 1982; 1: 195-204.

[30] Achermann P, Dijk D-J, Brunner DP, Borbély AA. A model of human sleep homeostasis based on EEG slow-wave activity: quantitative comparison of data and simulations. Brain Res Bull 1993; 31: 97-113.

[31] Borbély AA, Baumann F, Brandeis D, Strauch I, Lehmann D. Sleep deprivation: effect on sleep stages and EEG power density in man. Electroencephalogr Clin Neurophysiol 1981; 51: 483-93.
[32] Dijk DJ, Beersma DG, Daan S. EEG power density during nap sleep: reflection of an hourglass measuring the duration of prior wakefulness. J Biol Rhythms 1987; 2(3): 207-19.

[33] Dijk DJ, Brunner DP, Beersma DG, Borbely AA. Electroencephalogram power density and slow wave sleep as a function of prior waking and circadian phase. Sleep 1990; 13(5): 430-40.

[34] Drewes AM, Nielsen BM, Taagholt SJ, Bjerregard K, Sevendsen L, Gade J. Sleep intensity in fibromyalgia: focus on the microstructure of the sleep process. Br J Rheumatol 1995; 34: 629-35.

[35] Hori A. Sleep characteristics in twins. Jpn J Psychiatry Neurol 1986; 40(1): 35-46.

[36] Linkowski P. EEG sleep patterns in twins. J Sleep Res 1999; 8(suppl 1): 11-3.

[37] Hrubec Z, Robinette CD. The study of human twins in medical research. N Engl J Med 1984; 310(7): 435-41.

[38] Robins LN, Helzer JE. Diagnostic Interview Schedule (DIS): version III-A. Department of Psychiatry, Washington University School of Medicine 1985.

[39] American Psychiatric Association. Diagnostic and Statistical Manual of Mental Disorders $3^{\text {rd }}$ rev. ed. Washington DC: Trade paperback publishers 1987.

[40] Eisen S, Neuman R, Goldberg J, Rice J, True W. Determining zygosity in the Vietnam era twin registry: an approach using questionnaires. Clin Genetics 1989; 35: 423-32.

[41] Torgersen S. The determination of twin zygosity by means of a mailed questionnaire. Acta Genetics Med Gemellology (Roma) 1979; 28: 225-36.

[42] Keith L, Machin G. Zygosity testing: current status and evolving issues. J Reprod Med 1997; 42: 699-707.

[43] Press WH, Flannery BP, Teukolsky SA, Vetterling WT. Numerical Recipes Pascal. New York: Cambridge University Press 1989.

[44] Feinberg I, Fein G, Floyd TC. Systematic trends across the night in human sleep cycles. Psychophysiology 1979; 16: 283-91.

[45] Armitage R, Landis C, Hoffmann R, et al. The impact of a 4-hour sleep delay on slow wave activity in twins discordant for chronic fatigue syndrome. Sleep 2007; 30(5): 657-62.

[46] Borbély A. Secrets of sleep. New York, NY: Basic Books, Inc 1984.

[47] Armitage R. The effects of antidepressants on sleep in patients with depression. Can J Psychiatr 2000; 45: 803-9.

[48] Moldofsky H, Scarisbrick P, England R, Smythe H. Musculoskeletal symptoms and non-REM sleep disturbance in patients with "fibrositis syndrome" and healthy subjects. Psychosom Med 1975; 37 : $341-51$.

(C) Hoffmann et al.; Licensee Bentham Open.

This is an open access article licensed under the terms of the Creative Commons Attribution Non-Commercial License (http://creativecommons.org/licenses/by-nc/3.0/) which permits unrestricted, non-commercial use, distribution and reproduction in any medium, provided the work is properly cited. 